\title{
The solar chromosphere at high resolution with IBIS
}

\section{New insights from the Ca II $854.2 \mathrm{~nm}$ line ${ }^{\star}$}

\author{
G. Cauzzi ${ }^{1}$, K. P. Reardon ${ }^{1}$, H. Uitenbroek ${ }^{2}$, F. Cavallini ${ }^{1}$, A. Falchi ${ }^{1}$, R. Falciani ${ }^{3}$, \\ K. Janssen ${ }^{1}$, T. Rimmele ${ }^{2}$, A. Vecchio ${ }^{1}$, and F. Wöger ${ }^{2,4}$ \\ 1 INAF - Osservatorio Astrofisico di Arcetri, 50125 Firenze, Italy \\ e-mail: gcauzzi@arcetri.astro.it \\ 2 National Solar Observatory, PO Box 62, Sunspot NM 88349, USA \\ 3 Dipartimento di Astronomia, Università di Firenze, 50125 Firenze, Italy \\ ${ }^{4}$ Kipenheuer Institute für Sonnenphysics, 79104 Freiburg, Germany
}

Received 10 September 2007 / Accepted 30 November 2007

\begin{abstract}
Context. The chromosphere remains a poorly understood part of the solar atmosphere, as current modeling and observing capabilities are still ill-suited to investigating its fully 3-dimensional nature in depth. In particular, chromospheric observations that can preserve high spatial and temporal resolution while providing spectral information over extended fields of view are still very scarce.

Aims. In this paper, we seek to establish the suitability of imaging spectroscopy performed in the Ca II $854.2 \mathrm{~nm}$ line as a means of investigating the solar chromosphere at high resolution.

Methods. We utilize monochromatic images obtained with the Interferometric BIdimensional Spectrometer (IBIS) at multiple wavelengths within the CaII $854.2 \mathrm{~nm}$ line and over several quiet areas. We analyze both the morphological properties derived from narrow-band monochromatic images and the average spectral properties of distinct solar features such as network points, internetwork areas, and fibrils.

Results. The spectral properties derived over quiet-Sun targets are in full agreement with earlier results obtained with fixed-slit spectrographic observations, highlighting the reliability of the spectral information obtained with IBIS. Furthermore, the very narrowband IBIS imaging reveals very clearly the dual nature of the Ca II $854.2 \mathrm{~nm}$ line. Its outer wings gradually sample the solar photosphere, while the core is a purely chromospheric indicator. The latter displays a wealth of fine structures including bright points akin to the $\mathrm{Ca} I I \mathrm{H}_{2 V}$ and $\mathrm{K}_{2 V}$ grains, and as fibrils originating from even the smallest magnetic elements. The fibrils occupy a large fraction of the observed field of view, even in the quiet regions, and clearly outline atmospheric volumes with different dynamical properties, strongly dependent on the local magnetic topology. This highlights how 1D models stratified along the vertical direction can provide only a very limited representation of the actual chromospheric physics.

Conclusions. Imaging spectroscopy in the Ca II $854.2 \mathrm{~nm}$ line currently represents one of the best observational tools for investigating the highly structured and highly dynamical chromospheric environment. A high-performance instrument such as IBIS is crucial in achieving the necessary spectral purity and stability, spatial resolution, and temporal cadence.
\end{abstract}

Key words. Sun: chromosphere - Sun: magnetic fields - instrumentation: high angular resolution - instrumentation: interferometers

\section{Introduction}

The chromosphere embodies the transition between the photosphere and the corona, two regions dominated by vastly different physical regimes. In particular, it is within the chromosphere that the plasma $\beta$, the ratio of plasma kinetic pressure to magnetic pressure, falls below unity, signaling a shift from hydrodynamic to magnetic forces as the dominant agent in the structuring of the atmosphere. As ably described in the review of Judge (2006), the combined effects of magnetic field guidance and small-scale gas thermodynamics lead to the impressive amount of fine structures (jets, spicules, fibrils, mottles, etc.) that uniquely characterize this part of the solar atmosphere. Such fine structure represents a formidable challenge even to the most modern instrumentation, as its study requires high spectral resolution, as needed to resolve line profiles encoding large gradients or discontinuities

$\star$ Two movies are only available in electronic form at http://www . aanda.org (e.g. the shocks of Carlsson \& Stein 1997), combined with extremely high temporal and spatial resolution (see e.g. the recent observations by van Noort \& Rouppe van der Voort 2006).

Among the chromospheric diagnostics accessible to groundbased observations, the $\mathrm{Ca}$ II $\mathrm{H}$ and $\mathrm{K}$ resonance lines have been used most extensively (see e.g. the review of Rutten \& Uitenbroek 1991). These lines are the broadest lines in the visible spectrum, sampling a wide range in formation height, and are the only visible lines that provide a direct indication of the chromospheric temperature rise with their $\mathrm{H}_{2}$ and $\mathrm{K}_{2}$ emission reversals. Because the lines originate from the ground state of a dominant ionization stage, they are mostly collisionally controlled in the lower chromosphere and sensitive to local temperature to a much greater degree than, say, the hydrogen Balmer lines. Of these, the $\mathrm{H} \alpha$ line in particular has also been widely exploited, especially following the development of the Lyot filter (Lyot 1933), which gave rise to the science of "solar cinematography", i.e. narrow-band imaging obtained at rapid 
cadence. Even though $\mathrm{H} \alpha$ spectra are difficult to interpret because of the complicated formation characteristics of this line, much of what we know about the chromosphere derives from the detailed $\mathrm{H} \alpha$ morphology observed through Lyot-style filters.

A somewhat neglected, but no less interesting, chromospheric diagnostics is represented by the Ca II infrared triplet $(\lambda=849.8,854.2,866.2 \mathrm{~nm}$, hereafter Ca II IRT). These lines originate in the transitions between the upper $4 p^{2} \mathrm{P}_{1 / 2,3 / 2}$ levels and the lower metastable $3 \mathrm{~d}^{2} \mathrm{D}_{3 / 2,5 / 2}$ levels. Transitions between the ground state $4 \mathrm{~s}^{2} \mathrm{~S}_{1 / 2}$ of Ca II and the same upper levels give rise to the $\mathrm{H}$ and $\mathrm{K}$ lines. Since the branching ratio, the ratio between the spontaneous emission coefficients of the $\mathrm{H}$ and $\mathrm{K}$ lines and those of the IRT lines, is about $A_{\mathrm{HK}} / A_{\mathrm{IRT}} \sim 15$, most photons emitted in the IRT result from excitation in the $\mathrm{H}$ and $\mathrm{K}$ lines, leading to a very similar temperature sensitivity for both sets of lines. In addition, the $3 \mathrm{~d}^{2} \mathrm{D}_{3 / 2,5 / 2}$ are metastable (i.e., there are no allowed electric dipole transitions with the $4 \mathrm{~s}{ }^{2} \mathrm{~S}_{1 / 2}$ ground state), so that these levels can only be populated from below by collisional excitation, strengthening the sensitivity of the IRT to local temperature even more.

First detected in the 1878 eclipse spectrum by Young (Eddy 1973), the CaII IRT has remained essentially ignored in solar observational work for nearly a century. From the 1960's and up through the mid-1980's, it was occasionally employed, in particular in multi-line spectrographic studies (among others, Pasachoff et al. 1968; Linsky et al. 1970; Mein 1971; Shine \& Linsky 1972; Beckers et al. 1972; Shine \& Linsky 1974; Lites 1984). Spurred by the availability of CCD detectors with high sensitivity in this wavelength range, the past decade has instead seen a rapid growth of observational studies adopting these lines both for solar and cool star research; we refer the reader to Socas-Navarro et al. (2006), Uitenbroek (2006b), Tziotziou et al. (2006), Uitenbroek et al. (2006), and Pietarila et al. (2007b) for recent examples of solar studies, and to Chmielewski (2000), and Andretta et al. (2005) for the stellar case.

Despite the fact that near infrared wavelengths afford both a higher photon flux and a reduced terrestrial atmospheric disturbance (with respect to the wavelengths of $\mathrm{H}$ and $\mathrm{K}$ lines), highresolution solar observations of the Ca II IRT are still scarce. The great majority of the previous observations were in fact conducted with fixed-slit spectrographs, either performing an area raster scan, requiring long repetition times and thus compromising the temporal resolution (Fleck et al. 1994; Uitenbroek 2006b; Pietarila et al. 2007b) or keeping the slit at a fixed position (Deubner \& Fleck 1990; Pietarila et al. 2007b; Langangen et al. 2008). The latter strategy allows for a high temporal cadence, but makes it difficult to precisely follow the small chromospheric structures, often of magnetic origin, that are moved away from the slit either by atmospheric turbulence or solar evolution. A more efficient observational strategy is provided by the multichannel subtractive double pass technique (MSDP, Mein 1991, 2002). The MSDP is in fact one of the few examples of an integral field unit (IFU) device being used for optical solar observations, with the unique capability of acquiring truly simultaneous spectra over an extended field of view (FOV). However, for the case of the Ca II $854.2 \mathrm{~nm}$ line, observations are usually limited to a spectral range of about $\pm 40 \mathrm{pm}$ from the line core (e.g. Tziotziou et al. 2002), thus possibly neglecting important information related to strong variations encoded in the extended line profile. Moreover, the usable FOV represents a trade-off with the spectral coverage and resolution and is generally limited to only a few arcsec in one of the spatial directions.

In this framework, we introduce here new high- resolution observations of the Ca II $854.2 \mathrm{~nm}$ line obtained in a variety of solar structures with the Interferometric BIdimensional Spectrometer (IBIS, Cavallini 2006), installed at the Dunn Solar Telescope of the US National Solar Observatory. While not an IFU proper, IBIS is a high-performance instrument that combines most of the advantages of a full spectroscopic analysis, usually obtained with single-slit spectrographs, with the high spatial resolution, high temporal cadence, and the large FOV typical of filter instruments. Such characteristics are necessary to obtain new insights into the structure and dynamics of the chromosphere (see for example Vecchio et al. 2007b; Cauzzi et al. 2007). Although other instruments similar to IBIS are currently operative, such as TESOS (Tritschler et al. 2002) and the new Göttingen Fabry-Perot system (Puschmann et al. 2006), no other imaging spectrometer is presently able to access the wavelengths of the Ca II IRT.

\section{Instrumental characteristics}

IBIS is a tunable narrowband filter whose main components are two air-spaced, $50 \mathrm{~mm}$ diameter Fabry-Perot interferometers (Cavallini 2006; Reardon \& Cavallini 2007). IBIS operates in the spectral range $580-860 \mathrm{~nm}$ and can provide quasimonochromatic images at any wavelength in that range by suitable tuning of the interferometers. However, because of the periodic nature of the spectral transmission profile, the analysis of any given spectral line requires the use of a prefilter of $0.3-0.5 \mathrm{~nm} F W H M$, which isolates the central transmission order. Currently a prefilter for the Ca II $854.2 \mathrm{~nm}$ line of the IRT is available for IBIS. The filter was provided by Barr Associates, with an FWHM of $0.46 \mathrm{~nm}, 35 \%$ transmission, centered at $854.25 \mathrm{~nm}$. The central wavelength of the filter is slightly offset to the red from the nominal central wavelength of the Ca II $854.2 \mathrm{~nm}$ line to allow the filter to be tilted in the beam to avoid reflections. This also permits the passband to be minimally tuned in a range of approximately $\pm 0.1 \mathrm{~nm}$ from the central wavelength. We note also that, in the case of broad lines such as the CaII IRT, the limited prefilter passband effectively prevents the sampling of the line all the way to the continuum, as shown in the upper panel of Fig. 1.

The instrumental spectral transmission of IBIS has been accurately calculated and the $F W H M$ at $854 \mathrm{~nm}$ was determined to be 4.4 pm (Reardon \& Cavallini 2007). The spectral purity of the instrumental profile is quite high, with over $95 \%$ of the total transmission coming from within a range of $\pm 8 \mathrm{pm}$ around the peak of the profile and spectral parasitic light of only $1.5 \%$, arising from the repeating secondary orders of the transmission profile. The lower panel of Fig. 1 shows how this is accomplished with the combination of two interferometers and the prefilter, which strongly suppresses the secondary maxima in the Fabry-Perots transmission.

Piezoelectric tuning allows the instrumental profile to be positioned at multiple wavelengths within a given line, making it possible to acquire full spectral information over the $80^{\prime \prime}$ diameter circular FOV of the instrument. The Ca II $854.2 \mathrm{~nm}$ line is typically sampled at $15-30$ wavelength positions, depending on the scientific requirements (see following sections). Given the current rate of acquisition (2.5-4 frames s $\left.{ }^{-1}\right)$, this translates into an interval of 5-12 s to perform a full sampling of the line. A planned upgrade for the camera system in the near future may improve this rate by a factor of $2-4$.

To obtain meaningful spectral information from sequential images at high spatial resolution, it is necessary that the location of the structures remain stable during the spectral scanning of the line. The high-order adaptive optics system of the DST 
Table 1. Summary of the Ca II $854.2 \mathrm{~nm}$ observations described in the paper.

\begin{tabular}{lllllllllll}
\hline \hline Date & Target & Location & $\Delta x\left({ }^{\prime \prime}\right)$ & $t_{\exp }(\mathrm{ms})$ & $\delta \lambda(\mathrm{pm})$ & $\Delta \lambda(\mathrm{nm})$ & $N$ & $\delta t(\mathrm{~s})$ & $\Delta t(\mathrm{~s})$ & Duration $(\mathrm{min})$ \\
\hline 20040531 & quiet Sun & disk center & 0.16 & 25 & $8-16$ & {$[-0.12,0.16]$} & 27 & 7 & 19 & 55 \\
20040602 & quiet Sun & disk center & 0.16 & 25 & $8-16$ & {$[-0.12,0.16]$} & 27 & 7 & 19 & 55 \\
20051001 & small pore & disk center & 0.082 & 50 & 4 & {$[-0.07,0.13]$} & 35 & 700 & - & - \\
\hline
\end{tabular}

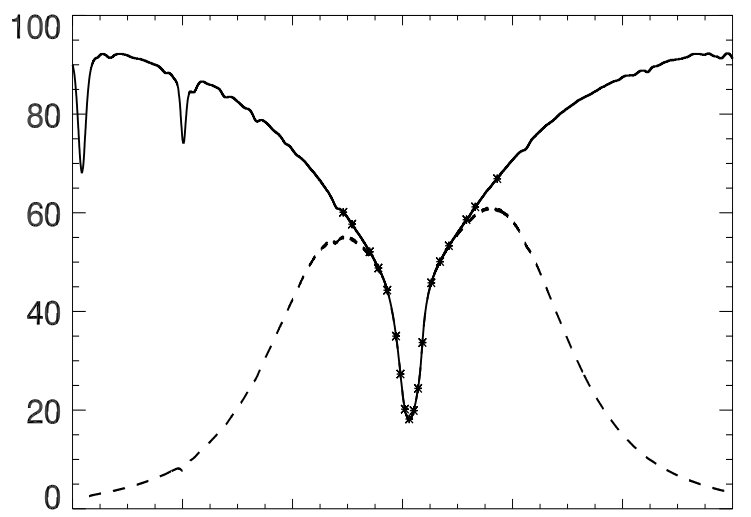

$\begin{array}{llllll}853.6 & 853.8 & 854.0 & 854.2 & 854.4 & 854.6\end{array}$

Wavelength $(\mathrm{nm})$

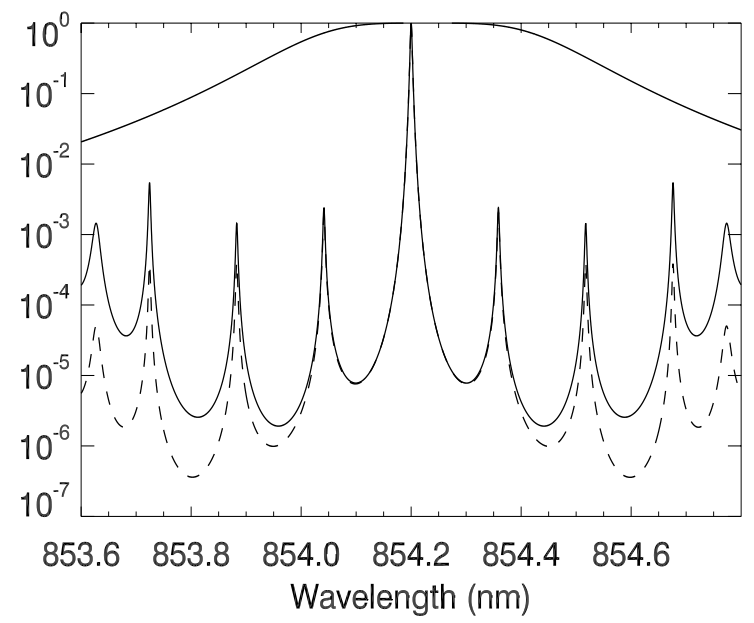

Fig. 1. Top panel: the solid line displays the Ca II $854.2 \mathrm{~nm}$ atlas profile, while the dashed line shows the effects of the $0.46 \mathrm{~nm}$ FWHM prefilter utilized in IBIS observations. The actual maximum transmission of the prefilter is $35 \%$. The asterisks indicate the wavelengths of the images in Figs. 2 and 3. Bottom panel: normalized prefilter profile (solid, upper curve) and periodic transmission profile of the combined Fabry Perot's (solid, multiple peaks curve). Note the logarithmic scale. The dashed line indicates the effects of the prefilter on the FPs transmission profile, i.e. the large suppression of the secondary maxima of the curve, as needed to reduce the spectral parasitic light.

(Rimmele 2004) is routinely used with IBIS and greatly aids in stabilizing the image. To remove residual image motion, especially at increasing distances from the AO lock point, we obtain a broadband ("white light") reference image strictly simultaneously with each narrowband image. These reference images are obtained through $10 \mathrm{~nm}$ wide filters and, during the relatively short time needed for a spectral scan, show essentially the same scene (as compared to the spectral images where the structures change dramatically during the scan). They are then normally used to destretch the images in both channels or for more advanced image reconstruction techniques, such as deconvolution of the narrowband channel image supported by MOMFBD (van Noort et al. 2005) or speckle imaging (Wöger 2006).
Finally, the spatial scale of the IBIS images is set at 0.'082/pixel. With the use of the procedures described above, it is thus possible to exploit the periods of good to excellent seeing occurring at the DST up to the nominal diffraction limit of the telescope of $\lambda / D=0.23$ at $854.2 \mathrm{~nm}$.

\section{Observations}

Table 1 lists the example Ca II $854.2 \mathrm{~nm}$ data presented in this paper. Date is expressed in yyyymmdd; $\Delta x$ is the pixel spatial scale; $t_{\exp }$ is the exposure time; $\delta \lambda$ is the spectral sampling step, while $\Delta \lambda$ is the total sampled range; $N$ is the number of wavelength points; $\delta t$ is the scan time, while $\Delta t$ is the cadence of the observations. For the case of the Ca II $854.2 \mathrm{~nm}$ line, often the sampling is coarser in the extended wings with respect to the core.

The data listed in Table 1 were acquired mostly in quiet target areas. We point out, however, that IBIS observations in this spectral line are well-suited also to studies of solar activity. On the one hand, the $80^{\prime \prime}$ diameter FOV is able to accommodate large portions of active regions (or even full small ones), thus overcoming a typical problem of spectrographic observations. On the other, the ability to obtain spectral profiles in just a few seconds (ten or less) allows detailed study of the dynamics of rapid impulsive events, such as reconnection-driven explosive events or small flares occurring in the solar chromosphere.

The sequence of monochromatic ${ }^{1}$ images of Fig. 2 shows a scan acquired on 2004 May 31. The actual wavelengths of observation are indicated in the panels as offsets from the nominal line core. The target was a quiet area at disk center with mostly unipolar, weak network elements within the FOV. The whole region was positioned at the edge of an equatorial coronal hole, as seen from EIT $171 \AA$ full disk images. The first panel shows the corresponding white-light image, while the last one displays a cotemporal, high-resolution MDI map of the longitudinal magnetic flux.

Figure 3 shows the data acquired on 2004 June 2, with the same setup as 2004 May 31. The target was again a quiet area at disk center, this time roughly encompassing a full supergranule surrounded by some enhanced, bipolar network. The whole region appeared as a fading coronal bright point in the EIT $171 \AA$ full disk images, where occasional bouts of smallscale activity were observed. Numerous bright points, as well as several rapidly evolving small pores, can be distinguished in the broadband image in the regions of the network, testifying to a stronger magnetic flux as seen in the MDI panel. This dataset was analyzed in Vecchio et al. (2007b) and in part in Janssen \& Cauzzi (2006).

Figure 4 shows the target region of 2005, Oct. 1, encompassing a small pore $\left(3^{\prime \prime}-4^{\prime \prime}\right.$ diameter) within a region of weak plage near disk center. This dataset was acquired to test the procedure

\footnotetext{
1 Due to the classical mounting of the interferometers, the spectral passband of IBIS experiences a radial, wavelength-dependent, blueshift within the FOV. This effect has been removed from all the images displayed in this paper.
} 


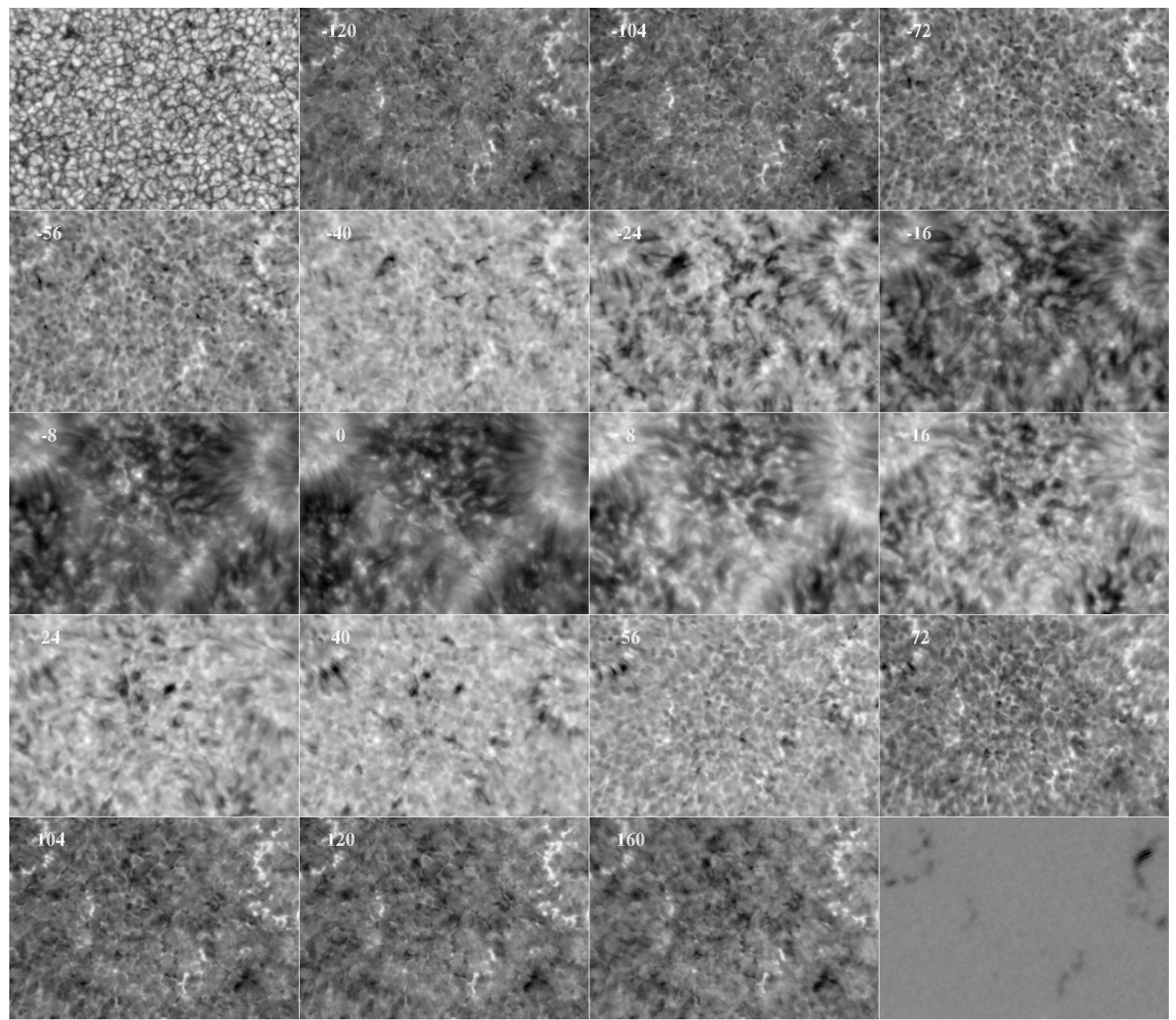

Fig. 2. Ca II $854.2 \mathrm{~nm}$ data acquired on 2004 May 31. Wavelength runs from left to right, top to bottom, as indicated in the panels, with offsets in $\mathrm{pm}$ from the nominal line core. The figure shows a cutout of $64^{\prime \prime} \times 41^{\prime \prime}$ of the full circular FOV, originally $80^{\prime \prime}$ in diameter. Each panel was normalized for optimum display. The topmost left panel shows the cospatial, cotemporal broadband image (acquired at $710 \mathrm{~nm}, F W H M=10 \mathrm{~nm}$ ). The bottom right panel gives the cotemporal HR MDI map, scaled between \pm 500 G. The intensity in the far wings of the Ca II $854.2 \mathrm{~nm}$ line is strongly sensitive to the presence of magnetic structures (cf. Leenaarts et al. 2006, and Sect. 5.2).

for performing post-facto image reconstruction using the speckle code of Wöger (2006), hence 50 repeated exposures were obtained at each wavelength point, resulting in a long total acquisition time. The top panel displays the reconstructed image at about $60 \mathrm{pm}$ in the blue wing, while the bottom one gives the image at $20 \mathrm{pm}$, again in the blue wing. A movie displaying a cotemporal G-band frame, together with the tuning of the IBIS passband through the blue wing of the line is available in the online version of this paper $^{2}$. This data clearly illustrates the wealth of fine structure information the Ca II IRT can provide when observed at high resolution (see also Fig. 11 in Rutten 2007).

\section{Line formation}

The most striking characteristics of the narrowband sequences of Figs. 2-4 is the dramatic change in scenery when the instrumental passband moves from the wings towards the core of the

${ }^{2}$ Movie 1, available at http: //www . aanda.org line - the latter displaying swarms of fibrillar structures usually associated only with $\mathrm{H} \alpha$ core diagnostics. The "critical wavelength", at about $30-40 \mathrm{pm}$ from the core, corresponds to the inflection point in the line where the steep inner wings give way to the much shallower outer wings (see the profiles in Fig. 1).

The sharp knees in the line profile mark the transition from LTE absorption line in the photospheric outer wings to the nonLTE chromospheric absorption line core. This transition is a reflection of two effects in the line source function and in the line opacity. In the photosphere, where LTE applies, the line source function decreases with height with the electron temperature, giving rise to a slow decrease in line-wing intensity towards line center. Because of their LTE formation, the wings of the $854.2 \mathrm{~nm}$ line are indeed used as accurate temperature diagnostics in solar type stars (e.g., Linsky et al. 1979; Smith \& Drake 1987). In the chromospheric layers, collisional excitation and de-excitation lose dominance to radiative transitions as the main population mechanisms in the line. Photon losses to outer space propagate inward because of the scattering in the line that results. With these losses the radiation field in the line can no 


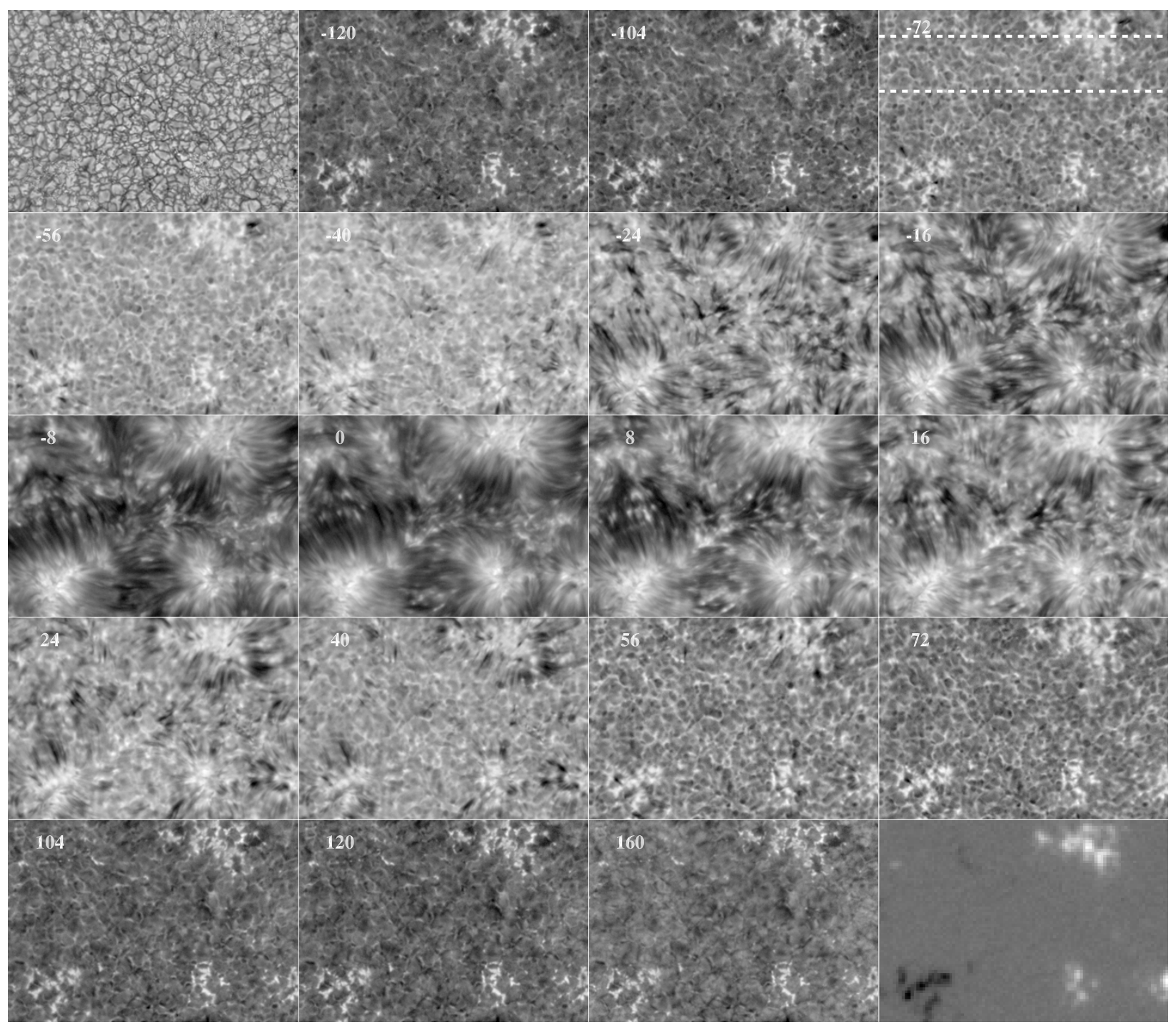

Fig. 3. Same as Fig. 2, for 2004 June 2 data. In the speckle-reconstructed broadband image many bright points can be appreciated in the network areas. The MDI map shows a strong, bipolar network (image scaled between $\pm 500 \mathrm{G}$ ). Note how the fibrillar structures originating from the network elements, very visible in the inner wings and core images, extend over a substantial fraction of the FOV, different from what occurs in the area depicted in Fig. 2. The two dashed lines in the upper-right panel indicate the positions where the slit spectra of Fig. 6 have been obtained.

longer maintain the upper level population of the line to LTE levels, and the line source function drops steeply with height.

The sharp transition between these two regimes results from a gap in the line opacity around the temperature minimum. Since the lower levels of the IRT are the metastable $3 \mathrm{~d}^{2} \mathrm{D}_{3 / 2,5 / 2}$ levels, their population in low-temperature regions is reduced compared to the ground-level population. This is a small effect because the excitation energy of the D levels is only $1.5 \mathrm{eV}$ (e.g. much smaller than the $10 \mathrm{eV}$ of the hydrogen $\mathrm{H} \alpha$ line for which the gap in line opacity is therefore much more pronounced). Nevertheless, because of this gap in line opacity, the formation height quickly shifts from photospheric to chromospheric for small wavelength differences around the knees of the line. This quick transition in formation height is obvious in the line intensity contribution function, displayed in Fig. 5 as a function of wavelength (on the horizontal axis) and height in the atmosphere (on the vertical axis). At wavelengths for which the height of $\tau_{\lambda} \sim 1$ corresponds to the height of the line opacity gap, the contribution function rises steeply with height, indicating that the line-intensity sensitivity quickly changes from photospheric to chromospheric (see also Uitenbroek 1989; Qu \& Xu 2002).
The very narrow spectral passband of IBIS makes it possible to clearly distinguish these two regimes directly in the imaging, with a rapid change with wavelength in the observed morphology. The outer wing images thus mostly display convective structures, only occasionally "contaminated" by fibrils that show up as thin dark strikes. The core images instead display mostly fibrillar, chromospheric structures that appear most evident in the blue or red inner wings depending on their line width and Dopplershift. Only in very quiet portions of the FOV are the fibrils not the predominant feature, and sporadic bright points with size on the order of 1 arcsec become visible in the core (see Sect. 5.3).

\section{Morphology}

\subsection{Outer wings - quiet photosphere}

As just discussed, the high-spatial resolution images obtained with IBIS in the outer wings of the Ca II $854.2 \mathrm{~nm}$ line are dominated by photospheric structures. In particular, the pattern of reverse granulation, i.e. a partial reversal of the contrast between 


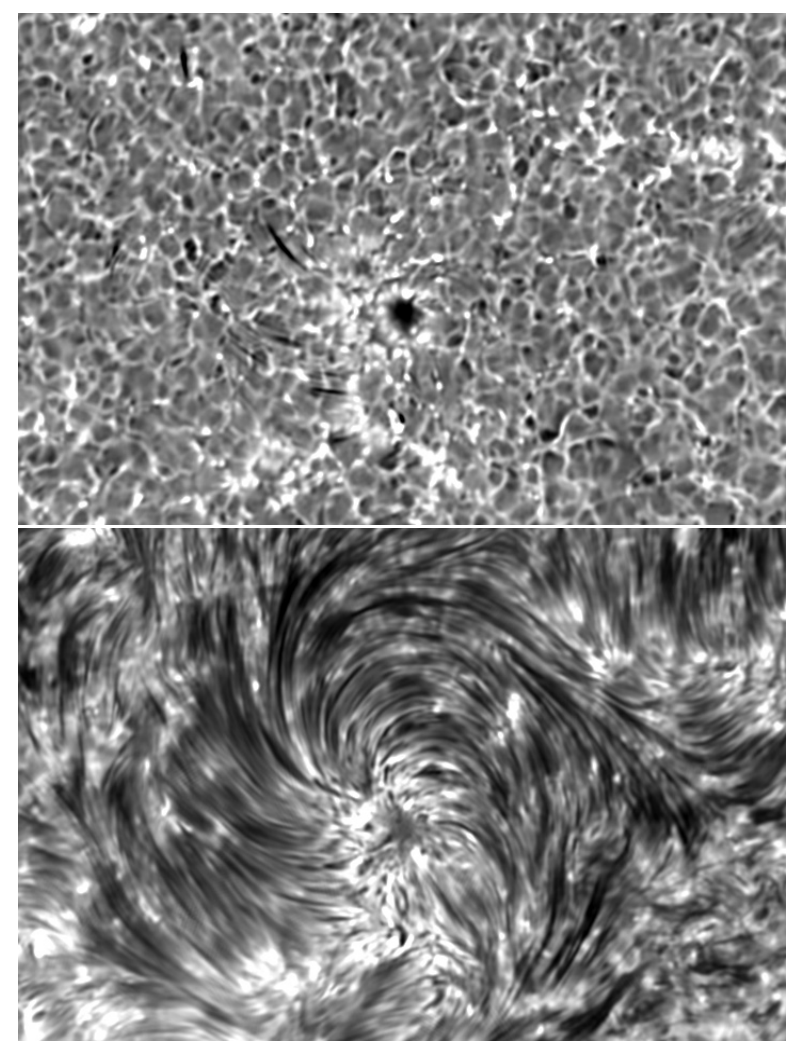

Fig. 4. Speckle-reconstructed sample images from the weak plage region of Oct. 1, 2005. Upper panel: image obtained at $65 \mathrm{pm}$ in the blue wing of the line. FOV is about $60^{\prime \prime} \times 40^{\prime \prime}$. Bottom panel: corresponding image at $20 \mathrm{pm}$ in the blue wing, obtained $200 \mathrm{~s}$ later. Images have been reconstructed applying the speckle code of Wöger (2006). See the online version for a movie of these data.

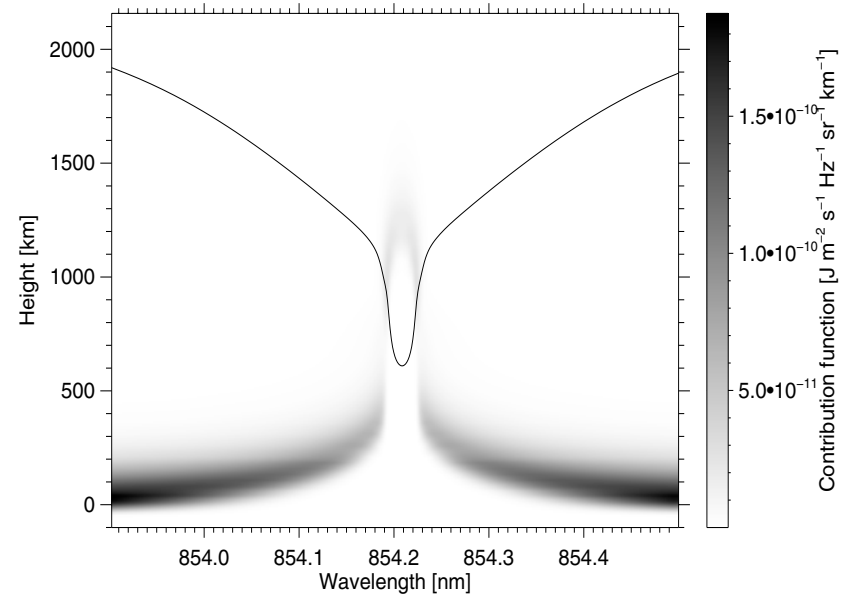

Fig. 5. Contribution function for Ca II 8542 computed in plane-parallel, hydro-static, average quiet-Sun atmosphere (Fontenla et al. 1993). Height zero refers to the level at which optical depth in the continuum at $500 \mathrm{~nm}$ is unity.

granules and intergranules with respect to the continuum (see, e.g. Rutten et al. 2004, and references therein), stands out very clearly within the quiet portions of the FOV. This is visible, for example, by comparing the white-light images of Figs. 2 and 3 and the panels at $-100 \mathrm{pm}$ (see also Janssen \& Cauzzi 2006), or, even more clearly, in the first images of Movie 1. Looking carefully at the wing images of Figs. 2 and 3, one can also notice a slight asymmetry between the blue and the red wings, with reversed granulation more prominent in the blue, at an equal distance from the line core. This is due to the effect of cross-talk between intensity and velocities in the convective structures, much like the case for monochromatic images acquired in the wings of typical photospheric lines (Janssen \& Cauzzi 2006).

As one moves farther out in the wings, reverse granulation gradually disappears, and the quiet areas only show a diffuse intensity without any obvious structure (compare e.g. the last panel of Fig. 3, at 160 pm from line core). Given the broader sampling of the line in the red (cf. Table 1), this effect is mostly visible in the redmost wavelengths displayed in the figures. As mentioned in Sect. 2, normal IBIS sampling does not reach continuum wavelengths. (Although technically feasible, the measures would be much noisier and affected by a high level of spectral parasitic light.) Hence, our observations do not go far enough away from the line core to see the normal granular structures of the low photosphere.

Using 3D radiative hydrodynamics simulations, Cheung et al. (2007) have recently offered the most comprehensive explanation of the horizontal structure of the photospheric temperature and of reverse granulation, in particular. The latter results from the interplay between cooling of granules rising and expanding within the optically thin layers of the photosphere and from radiative heating acting against it. The (horizontally averaged) layer at which the temperature contrast between granules and intergranules vanishes is positioned at about $150 \mathrm{~km}$ above the classical photospheric surface $\left(\tau_{500}=1\right)$, hence photospheric diagnostics originating above this height are bound to show evidence of the reversed temperature contrast with respect to the continuum granulation. This is indeed consistent with the wing observations presented above, when interpreted through the contribution function of Fig. 5: at about $0.1 \mathrm{~nm}$ from line core the layers contributing most to the intensity are above $\sim 150 \mathrm{~km}$, i.e. a level where reversed granulation is already present. Instead, at about $0.15 \mathrm{~nm}$ the intensity forms slightly lower, in intermediate layers where the granular contrast is considerably reduced.

\subsection{Outer wings - the magnetic photosphere}

Besides reverse granulation, the outer wing images very clearly display the location of magnetic structures, such as the plage or quiet network visible in Figs. 2-4. Given their large temperature sensitivity, the intensity in the outer wings basically maps the lower opacity, higher temperature, small-scale magnetic elements. Their visibility is further enhanced at some wavelengths by the reduced contrast of the solar granulation. The co-temporal MDI high-resolution magnetic maps for the case of 2004 May 31, and 2004 June 2, demonstrate that there is a oneto-one correspondence between bright wing structures and magnetic patches. Actually, the higher resolution IBIS images clearly reveal how single magnetic features visible in MDI are composed of several distinct structures of sub-arcsec size, both in the network and, possibly, the internetwork (Janssen et al. 2003).

The use of intensity proxies as a diagnostics for small-scale magnetic elements in the photosphere has been recently investigated by Leenaarts et al. (2006), by numerically synthesizing several spectral diagnostics in a snapshot of a 3D solar magnetoconvection simulation. They identify the outer wings of Ca II $854.2 \mathrm{~nm}$ as one of the best proxies (albeit at the price of a reduced spatial resolution with respect to the wings of $\mathrm{H} \alpha$ or $\mathrm{H} \beta$ ), consistently with the results shown here. Somewhat at odds with their results, however, we find that the intensity images at $-0.09 \mathrm{~nm}$ from the line core already show the reversed granulation pattern (as discussed in the previous section) rather than 
normal granulation. In our data magnetic structures are hence better identified in intensity images obtained farther out in the wings (up to the limit of our sampling, i.e. $0.16 \mathrm{~nm}$ from core), where the granulation contrast almost vanishes. This discrepancy should be further investigated by a more detailed comparison between simulations and-high quality observations.

\subsection{Core structures}

Narrow-band Ca II 854.2 snapshots, acquired around the line core wavelength in the quiet regions, display a clear segregation of the FOV in various components (see Figs. 2 and 3). The distinction is even clearer if one has access to the temporal dimension: a movie in the online version ${ }^{3}$ shows the evolution of the nominal Ca II $854.2 \mathrm{~nm}$ line core as observed over the full FOV and for the full duration of the observations of 2004, May 31 (55 min with a cadence of $19 \mathrm{~s}$ ). Although the seeing conditions were at times variable, and certainly worse than for the data of 2004 June 2, we chose this dataset since it provides a clearer picture of the quietest internetwork regions. In particular, an almost complete supergranular cell ( $\sim 28 \mathrm{Mm}$ in diameter) is visible in the central-right portion of the FOV.

The first feature that one discerns is the magnetic network, again outlined by clusters of small, bright elements that faithfully map the presence of photospheric magnetic elements. The appearance of the network elements in the core images is more diffuse with respect to the wings of the line (Sect. 5.2), owing to the lateral spread of the magnetic field with height. However, it must be pointed out that their spatial extension seems to be very dependent on the instantaneous seeing conditions, as clearly visible in the movie. As long known, the chromospheric network elements evolve relatively slowly, with single features persisting for several minutes, and they present very different spectral properties and dynamics from the rest of the FOV (Sect. 6).

In contrast, the internetwork portions of the FOV are seething with smaller bright features, all rapidly evolving and immersed in an otherwise very dark environment. These bright points reach the same intensity as the network points (about 1.5-2 times the average core intensity), but are more sharply defined as roughly circular areas with $1^{\prime \prime}-3^{\prime \prime}$ diameter, and evolve with timescales of 1-2 min. At their darkest, they reach about 0.75 times the average core intensity. Many of the points seem to reappear throughout the course of the observations, with a cadence of a few minutes, and about 10-20 of them are present at any given time within the cell interior. These properties are in complete analogy to the properties of the $\mathrm{H}_{2 V}$ and $\mathrm{K}_{2 V}$ "grains" (see the review of Rutten \& Uitenbroek 1991). The latter have been explained as due to shocks generated by acoustic waves, with frequencies slightly above the acoustic cutoff, originating in the photospheric layers and propagating upwards in the absence of magnetic fields (Carlsson \& Stein 1995, 1997). The radiativehydrodynamical model of Carlsson \& Stein indicates that indeed the shocks should also be very visible in quiet areas also in the Ca II $854.2 \mathrm{~nm}$ line (Pietarila et al. 2006) but, to our knowledge, they have never been convincingly observed in this spectral signature. IBIS observations thus open the way to a more complete study of the phenomenon, as both the spectral and spatial domains are accessible at once, with many statistics provided by large FOVs and the opportunity to analyze different magnetic topologies. Results of such an analysis will be presented in a forthcoming paper (Vecchio et al. 2007a).

${ }^{3}$ Movie 2, available at http: //www . aanda.org
The third prominent feature in the Ca II $854.2 \mathrm{~nm}$ core images is due to fibrils, which seem to originate from even the smallest magnetic elements (compare Movie 1). Their appearance is strongly reminiscent of the structures observed in $\mathrm{H} \alpha$ (see the recent high-resolution observations of Rouppe van der Voort et al. 2007), although we do not have any direct comparison here between the two spectral signatures. Much as for the $\mathrm{H} \alpha$ fibrils, the structures observed in Ca II 854.2 differ noticeably between active regions and the quiet Sun. More active regions harbor long and relatively stable fibrils, together with shorter dynamic fibrils (Fig. 4), while the quiet Sun displays both long mottles, connecting the stronger magnetic concentrations in the network (a clear example is in the lower half of the FOV of Fig. 3), and shorter ones, presumably outlining field lines that reach outward to the corona and interplanetary space. The structures visible in Movie 2 most probably belong to the latter category, as the FOV lies at the edge of a coronal hole.

Several of the fibrils characteristics are worthy of notice: first of all, they occupy a large fraction of the FOV, even in the quietest of instances. For example, in the region observed on 2004 May 31, they occupy between 30\% and 50\% of the "internetwork" area, while they become the dominant component in the weak plage region of Fig. 4. Second, their dynamic is completely different from the internetwork areas: looking at the movie, one is given the clear impression of material flowing from the network elements outwards, i.e. of predominant transversal motions with respect to the solar surface, as opposed to the mostly vertical dynamics of the bright points. We do not observe significant lateral swaying of the fibrils, although this may be due to the lower spatial and temporal resolution compared to Rouppe van der Voort et al. (2007), who had a 1 s cadence (compared to the $19 \mathrm{~s}$ in our data) and a spatial resolution nearing the diffraction limit of the Swedish Solar Telescope (SST, $0.17^{\prime \prime}$ at the $\mathrm{H} \alpha$ wavelength). Finally, in some instances, small bright points like those described above are visible within the fibrils. Whether because of physical lateral motions, or because of changes in the opacity of the structure, at times the fibrils let us glimpse the quiet atmosphere underneath.

\subsection{Fibrils in Ca II?}

The overwhelming presence of fibrils in the Ca II $854.2 \mathrm{~nm}$ core images was an initial surprise of the IBIS observations, as they had never been observed at this level of detail other than in the $\mathrm{H} \alpha$ core and inner wings. Earlier Ca II $854.2 \mathrm{~nm}$ spectroheliograms by Title (1966, Plates 16-18), as well as MSDP observations, probably did not reach the necessary spectral and spatial resolution to fully appreciate these characteristics, although MSDP data have shown the presence of arch filament systems in emerging flux regions (Mein et al. 2000).

A striking point is that fibrils are normally absent from $\mathrm{Ca}$ II $\mathrm{H}$ and $\mathrm{K}$ imaging, unless observed at very high spatial resolution in the core of the lines, when they might appear as bright, upright stalks in network areas (e.g. Rutten 2006, 2007). This has led to the (often unspoken) assumption that the latter lines sample a "different", possibly lower chromosphere than $\mathrm{H} \alpha$ and that the enhanced visibility of fibrils in $\mathrm{H} \alpha$ imaging is due to some peculiarities of the line formation, in particular its dependence on photoionization-recombination mechanisms.

Given the strong interconnection between $\mathrm{CaII} \mathrm{H}$ and $\mathrm{K}$ and the IRT formation discussed in the previous sections, why then does the Ca II 854.2 line display such a different "chromospheric" scenario? We believe that a large part of the problem lies not in the infrared line formation, but in the strong 
observational limitations that still affect $\mathrm{Ca}$ II $\mathrm{H}$ and $\mathrm{K}$ data. In other words, the issue is not why the Ca II 854.2 nm line displays the chromospheric fibrils, but why the $\mathrm{H}$ and $\mathrm{K}$ do not show them! Essentially, the chromospheric signal of the $\mathrm{Ca}$ II $\mathrm{H}$ and $\mathrm{K}$ lines is confined to the rather narrow core (with Doppler width of $10-15 \mathrm{~km} \mathrm{~s}^{-1}$, i.e. $\leq 20 \mathrm{pm}$ at $400 \mathrm{~nm}$ ), while most of the imaging is performed with broad filters with passbands in the range 30-100 pm. These strongly dilute the core signal, however, resulting in images that are heavily biased toward the wing, upper-photospheric signal. Scattered light and general photometric noise further degrade the signal of interest. Finally, even with these relatively broad filters, the low photon flux near the core of these very deep lines (due also to the low transmission of the filters) requires long exposures, which compounds the increased seeing-induced distortions at the shorter wavelengths. For these reasons only very bright chromospheric structures (for example those related to network points) can survive the smearing and still be distinguishable above the photospheric background in the "core" images (Rutten 2006). To prove our point better, a direct comparison of simultaneous Ca II K filtergrams and Ca II 854.2 IBIS data will be presented in a forthcoming work. Preliminary results have been reported in Reardon et al. (2007).

\section{Spectral structure}

The most attractive quality of $2 \mathrm{D}$ spectrometers lies in the possibility of obtaining sufficiently detailed spectral information over an extended FOV, in a short period with respect to the evolutionary times of the structures. We describe in this section some characteristics of the CaII $854.2 \mathrm{~nm}$ spectral profiles obtained from the IBIS observations presented above. We use the quiet Sun dataset obtained on 2004 June 2, which can be easily compared to earlier results.

\subsection{Spectra and average line profiles}

Figure 6 shows two "slit spectra" derived from two cuts along the horizontal direction of the data displayed in Fig. 3. The spectral axis is obtained by interpolating the sequence of monochromatic values for each pixel along the cut. Given the acquisition sequence, the time also runs with the wavelength, and an interval of about $7 \mathrm{~s}$ separates the bluemost from the redmost wavelengths. The left spectrum intersects several network points (i.e. between positions 40 and 50 along the slit) and fibrils, while the right one covers mostly quiet areas. The quality of the spectra is high and appears comparable to that of data acquired at high spatial resolution with normal spectrographs (see for example the spectra obtained in the Ca II $866.2 \mathrm{~nm}$ line at the Swedish Solar Telescope by Langangen et al. 2008). Numerous indications of the dynamics are visible in the two regions, but with very different characteristics. The left panel displays strong redshifts of the line in several pixels (e.g. around positions 40 and 52, which become progressively stronger within a couple of arcsec and probably involve dynamic fibrils (De Pontieu et al. 2007). In the right panel, instead, stronger blueshifts are visible (e.g. positions $12,25,33$ along the slit), which are less extended in the spatial direction and have more constant amplitude. These are related to the development of acoustic shocks in non-magnetic areas (Pietarila et al. 2006; Vecchio et al. 2007a).

In Fig. 7 we show a comparison between the Liege atlas and the average observed line profile, obtained by summing over the whole dataset (more than $2 \times 10^{7}$ individual profiles). The effects of the prefilter transmission (cf. Fig. 1) have been accounted for,
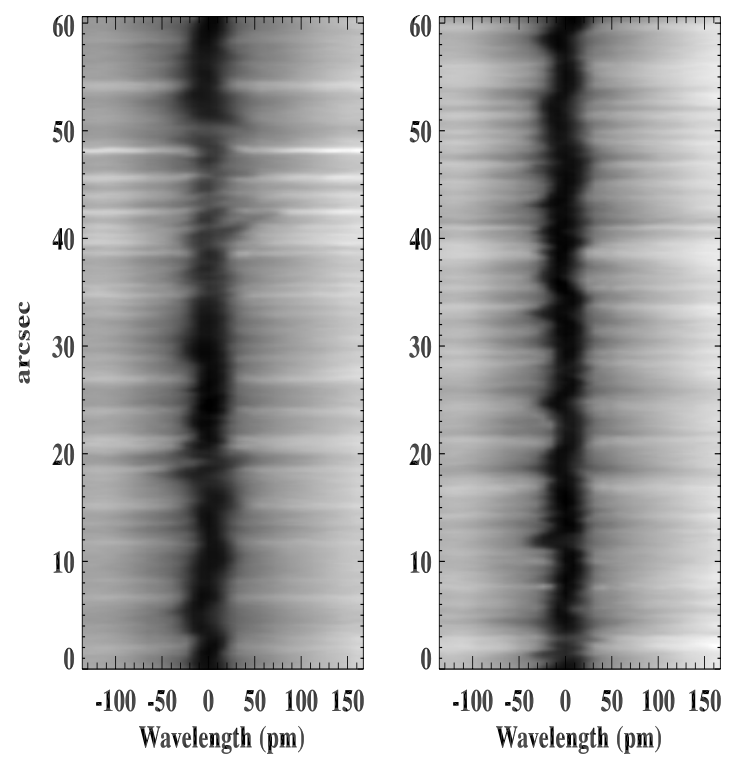

Fig. 6. "Slit" spectra obtained around the middle of the sequence of 2004 June 2, from two horizontal cuts of the data displayed in Fig. 3. The $x$-axis is the wavelength distance from the average line core position. The left spectrum corresponds to the upper slice across network areas, while the right one corresponds to the lower slice, and represents quiet internetwork areas.

and the wavelength offset adjusted so that the minimum of the average profile coincides with the atlas line-core position. The general agreement between the profiles is excellent, showing discrepancies of less than $2 \%$ of the continuum intensity around the inflection point in the wings (the greater difference at the blue limit of the observations is due to a telluric line). Differences of this amplitude are probably unavoidable, due both to slight residuals in the data calibration and to the intrinsic variability of the solar structures in time and space. Still, all the general characteristics of the atlas profile are reproduced well, including the asymmetry of the line core typical of the chromospheric lines ("inverse C-shape", Uitenbroek 2006b).

What kind of individual profiles enter into the average? To gain insight into this issue, we attempted a spatial classification for the three features discussed above: network, fibrils, and quiet internetwork. In this kind of analysis, an intensity threshold is often adopted in the definition of separate classes of pixels (e.g. Deubner \& Fleck 1990). However, as is clear from Sect. 5.3, the chromospheric scene is probably too complex to be properly described by a single static parameter. In particular, the fibrils, very prominent at the core wavelengths, appear here as both dark or bright with respect to the average contrast, making their recognition quite difficult. As a first attempt, we identified the three classes of pixels by adopting an intensity threshold applied to the temporal average of the observations, but using, for each feature, the intensity at the wavelength positions where they are most clearly separated from the background. First, the network has been defined as the bright pixels in the far wing of the line (at $+155 \mathrm{pm}$ from line core, having intensity above average plus one time the rms). This threshold clearly identifies all of the photospheric magnetic features, as seen e.g. in the cotemporal MDI magnetograms, which also have an obvious correspondence in the chromospheric line core intensity. Then, the fibrils and the internetwork areas were respectively defined as the dark and intermediate pixels in the inner wing intensity images $( \pm 20 \mathrm{pm}$ from line core), using the intensity values of the average plus 

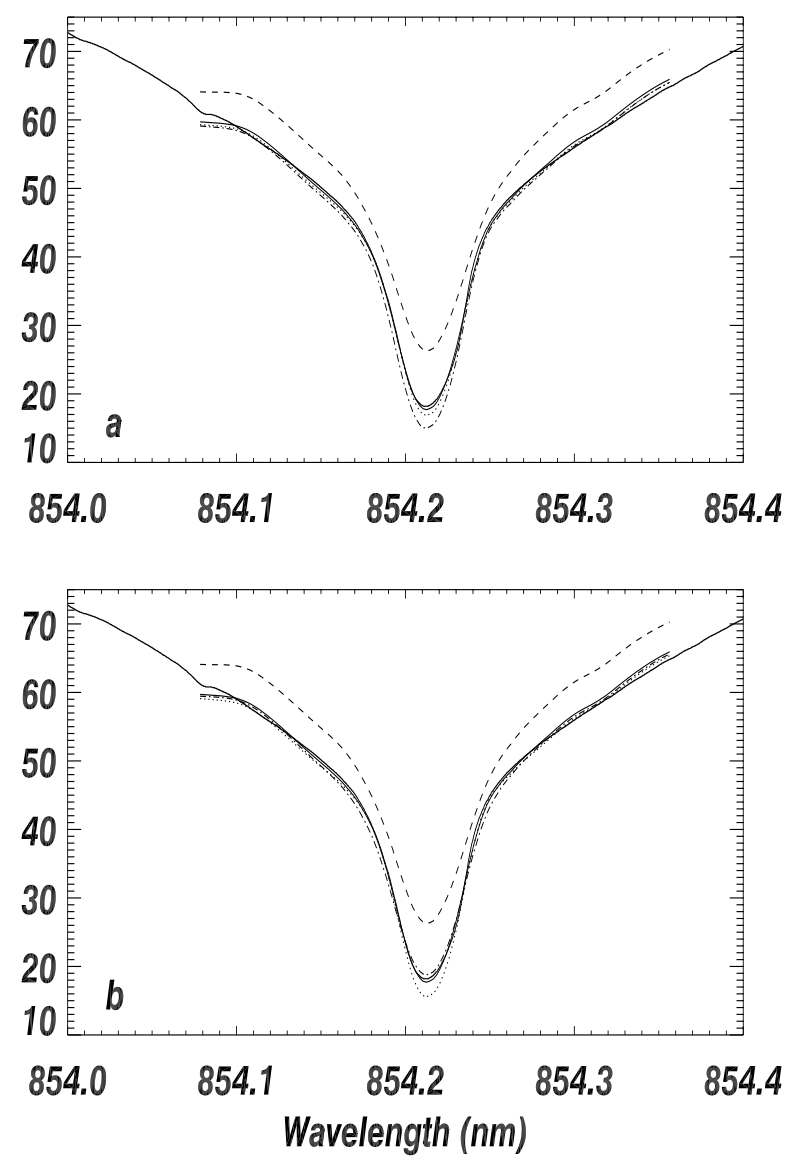

Fig. 7. Average line profiles for the data acquired on 2004 June 2. Panel a): the spectrum averaged over the whole dataset (thin solid line) is compared to the Liege atlas (thick solid). The effect of the prefilter transmission has been removed from the observations. The profiles averaged over different solar features defined by using an intensity threshold are also shown: network points (dashed), fibrils (dot-dashed), and internetwork (dotted). Panel b): as in panel a), but this time fibrils and internetwork areas were defined using dynamical properties.

or minus $1 / 3$ of the rms as our limits. These values separated the two classes of pixels clearly; in particular, they assured that there were no quiet pixels in the vicinity of network points, where fibrils supposedly originate. From our definition, the fractional area occupied was $9 \%$ (network points), 39\% (fibrils) and 35\% (internetwork), with the remaining pixels not assigned to any category. As expected, these unclassified pixels lie mostly around the magnetic elements. It is interesting to note that in this dataset the fractional area covered by fibrils is actually larger than the quiet Sun's, reflecting the closed magnetic topology of the region.

The averaged line profiles for the three classes are displayed in Fig. 7 as well. The network points average profile is much brighter than the reference one at all sampled wavelengths, is slightly redshifted (about $150 \mathrm{~m} \mathrm{~s}^{-1}$ ) and has a core asymmetry less pronounced than the average profile. The fibrils' profile is almost complementary to the network one: it is slightly blueshifted with respect to the average over the whole FOV (about $100 \mathrm{~m} \mathrm{~s}^{-1}$ ) and is deeper, with a core intensity about $3 \%$ lower (in units of the continuum intensity), while the overall wings remain close to the average. These same properties have been reported by Harvey (2005) in a study of "calcium circumfaculae" using Ca II 854.2 SOLIS data around active regions. He demonstrates how the areas of deeper, blueshifted Ca II 854.2 profiles coincided with the extended fibrilar regions observed in $\mathrm{H} \alpha$ core. Finally, the internetwork profile is only slightly deeper than the average, while most of the other profile characteristics remain very similar to the average over the whole FOV.

\subsection{Velocity distribution}

From the spectral profiles described above, we calculated lineof-sight velocities for each pixel in the FOV and each time step. To this end we used the Doppler shifts of the intensity minima of the spectral profiles, derived from second-degree polynomial fits of the line core. The zero has been defined as the spatio-temporal average position over the whole dataset. This approach has often been used for the case of quiet chromospheric dynamics (e.g. Deubner \& Fleck 1990), but one must be aware that the derived values might not be fully representative of the actual plasma velocities, especially in the presence of nonlinear phenomena such as shocks. In particular, we expect that our velocity determination might fail for a certain fraction of the pixels in the magnetic areas (2-3\% of the total pixels), for which we observe emission in the blue wing, similar to the case reported in Pietarila et al. (2007b). However, since we are only interested in giving general properties here, and given the overall small number of such pixels, we will keep this definition as a convenient parametric description of the dynamics of the system.

The upper panel of Fig. 8 displays the distribution of velocity values over the whole dataset. Positive values indicate red-shifts, i.e. downward motions. The distribution is skewed towards positive values, justifying the peak at the negative (upward) value of $-0.12 \mathrm{~km} \mathrm{~s}^{-1}$ since we defined the zero as the average value over the whole dataset. About $97 \%$ of the pixels are contained in the $\pm 3 \mathrm{~km} \mathrm{~s}^{-1}$ range, but outliers can reach large velocity values, above $10 \mathrm{~km} \mathrm{~s}^{-1}$. At these higher values the distribution becomes markedly asymmetric in the positive quadrant.

Decomposing the distribution into the three types of solar features described above, i.e. network, fibrils and internetwork areas (dotted), one sees that the various distributions have different widths, with the fibrils displaying the least number of extreme vertical flows, especially lacking strong downflows. The rms values of the distributions are, 1.5, 1.4, and $1.25 \mathrm{~km} \mathrm{~s}^{-1}$ for, respectively, network, internetwork areas, and fibrils. Furthermore, the enhanced positive lobe in the total velocity distribution is largely due to the network points, which account for about $40 \%$ of this signal despite representing less than $10 \%$ of the total pixels. Since neither fibrils nor quiet areas contribute much at the highest redshifts, the signal missing with respect to the whole FOV distribution must be due to the pixels left unclassified, positioned around the network. This further underlines the highly dynamic nature of the magnetic network elements, that for this enhanced flux region might be related to the dynamic fibrils often observed in plages (Hansteen et al. 2006; De Pontieu et al. 2007).

\subsection{Velocity power spectra}

To address the presence and relevance of periodicities in the Ca II 854.2 nm dynamics, the temporal evolution of line-of-sight velocities has been investigated via a standard Fourier analysis, performed separately on each spatial pixel. The Nyquist frequency of the observations is $26 \mathrm{mHz}$, and the frequency step is $0.33 \mathrm{mHz}$. Figure 8 (bottom panel) shows the resulting spatially averaged velocity power spectrum. It has a broad peak between $\sim 3$ and $7 \mathrm{mHz}$ (periodicities between 2.4 and $5 \mathrm{~min}$ ), 

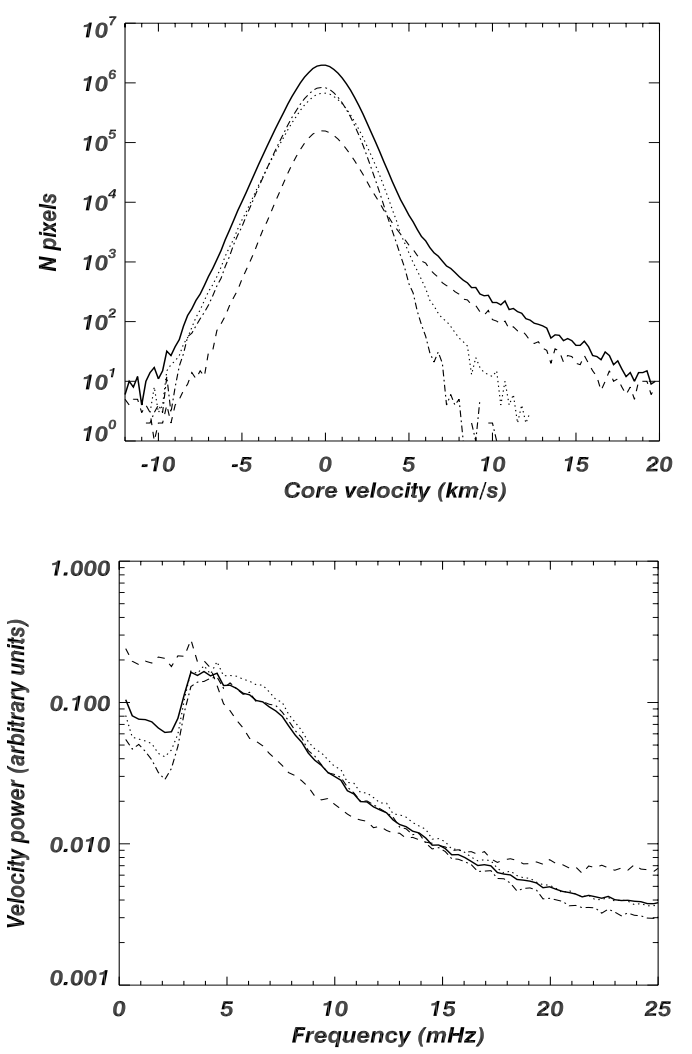

Fig. 8. Upper panel: line-of-sight velocity distribution for the dataset of 2004 June 2. Solid line: overall FOV and time (more than $2 \times 10^{7}$ values); dashed, dash-dotted, and dotted: distributions for, respectively, network points, fibrils, and internetwork. Bottom panel: velocity power spectra for the same features (symbols as upper panel). The curves represent an average over the corresponding pixels.

which indicates an almost equal contribution of both typical photospheric and chromospheric oscillatory signals to the average. This fully agrees with earlier results obtained using single-slit spectrographic observations on quiet solar regions (e.g. Noyes 1967; Fleck \& Deubner 1989). We note that the noise levels, determined by the accuracy of the core-fitting procedures, is comparable between these IBIS data and previous spectroscopic studies. (This accuracy was only achieved through the use of destretching techniques to correct for differential image motion.)

The power spectra relative to the three different atmospheric components defined above are also displayed in Fig. 8, using the same symbols as in Fig. 7. The curves are an average over the corresponding pixels; i.e., to recover the global power spectrum they must be weighted with their relative occurrence. They confirm several of the properties derived by earlier works, in particular that low-frequency oscillations are more prominent in network elements than in quiet areas, while periodicities of $3 \mathrm{~min}$ or shorter are essentially due to the quiet internetwork (see e.g. the Introduction of Deubner \& Fleck 1990). The network points display a clear peak at $\sim 3.5 \mathrm{mHz}$, but we cannot obviously identify any other maxima at lower frequencies (as claimed e.g. by Kalkofen 1997). The network power (per pixel element) at the $3.5 \mathrm{mHz}$ frequency is enhanced by about a factor of 2 with respect to the rest of the FOV, as reported in Lites et al. (1993). Known for some time, these latter properties have recently been related to the leakage of the dominant photospheric $p$-modes into higher atmospheric layers due to the lowering of the acoustic cutoff frequency in magnetic elements (Jefferies et al. 2006; Hansteen et al. 2006; Vecchio et al. 2007b).
Finally, from the plots of Fig. 8 we see that fibrils share many of the same properties of the quiet internetwork, with a somewhat reduced power at all frequencies (most evident between 5 and $8 \mathrm{mHz}$ ). Although in line with the results of Deubner \& Fleck (1990), this is a somewhat surprising result, given the very different properties of fibrils with respect to the "quiet" chromosphere, both in terms of morphology and dynamics (Sect. 5.3). This would also appear to contradict the results of Vecchio et al. (2007b) that, taking full advantage of the spatial resolution afforded by these data, showed how the fibrils are well-correlated with the "magnetic shadows", i.e. areas of reduced velocity power at frequencies above the acoustic cut-off, surrounding even the smallest magnetic elements (Judge et al. 2001). Armed with this knowledge, we show in panel b of Fig. 7 the same kind of average spectral profiles as are displayed in Fig. 7a, but this time the "fibrils" and "internetwork" areas are defined by classifying the spatial pixels on the basis of their oscillatory power rather than intensity. Over the frequency range $5.5-8.0 \mathrm{mHz}$, all pixels with a velocity power less than the mean of the whole FOV were defined as fibrils, while pixels with greater power than the average plus one rms were defined as quiet. These values clearly distinguish between the two different dynamical regimes. While a slight blueshift (about $70 \mathrm{~m} \mathrm{~s}^{-1}$ ) is still present in the fibrils' profile, its core intensity is now very close to the general average. In contrast, the internetwork profile becomes noticeably darker, as could be expected from the visual appearance of the quiet (i.e. non-magnetic) areas in Movie 1. We thus conclude that the adoption of a simple threshold of average intensity in order to distinguish between chromospheric structures can only be of limited value, as it does not effectively separate areas with significantly distinct chromospheric dynamics.

\section{Summary and conclusions}

We have presented an overview of the quiet solar chromosphere as observed in the Ca II $854.2 \mathrm{~nm}$ line at high spatial, spectral, and temporal resolution. This was made possible through the use of an advanced instrument, the Interferometric BIdimensional Spectrometer (IBIS, Cavallini 2006), installed at the NSO/Dunn Solar Telescope. To our knowledge, this is the first time that observations of this type and quality are reported for any chromospheric calcium line.

The stability and large throughput of the instrument, combined with the excellent performance of the high-order adaptive optics system available at the DST, make it possible to investigate the highly dynamic chromospheric environment while maintaining excellent spatial and spectral resolution, despite the obvious drawback (common to all filter-based instruments) of a sequential spectral acquisition. A comparison of the average properties of the spectral line profiles and of the global dynamics over a quiet target area with earlier results, obtained with classical fixed-slit spectrographic techniques, indeed demonstrates that the spectral information provided by IBIS is fully reliable and that it achieves a quality on a par with those of dispersion-based measurements.

The monochromatic images acquired with the very narrow spectral passband and high spatial resolution of IBIS (Figs. 2-4) display the "dual nature" of the Ca II $854.2 \mathrm{~nm}$ line with amazing clarity. In fact, much as for the $\mathrm{Ca}$ II $\mathrm{H}$ and $\mathrm{K}$ resonance lines, the broad wings of the Ca II $854.2 \mathrm{~nm}$ gradually sample the solar photosphere and provide a clear temperature diagnostics for these layers (Shine \& Linsky 1972, 1974). The core is instead a purely chromospheric indicator. Narrow-band imaging highlights these characteristics by showing a dichotomy 
of small-scale features at the different wavelengths. The outer wings clearly display phenomena of convective origin (such as the onset of reversed granulation) and the presence of bright magnetic elements. In contrast, the core images show highly dynamic small-scale bright "grains" akin to the $\mathrm{H}_{2 V}$ and $\mathrm{K}_{2 V}$ grains and fibrillar structures originating in small-scale magnetic structures and outlining the local magnetic topology. The nearly ubiquitous presence of these structures, particularly around the network magnetic elements, indicates that, even at these lower chromosphere heights, the atmosphere is already highly structured by the pervasive magnetic fields, consistent with the structures commonly seen in $\mathrm{H} \alpha$. The relative lack of fibrilar structures in observations of the $\mathrm{Ca}$ II $\mathrm{H}$ and $\mathrm{K}$ line images is probably due to the broader filters typically used (30-300 pm $F W H M$, the last being the width of the filter used on Hinode), which tend to mix the signal from vastly different regions of the atmosphere.

The demonstrated high spectral purity, imaging capabilities, and temporal stability of IBIS open up many possibilities in terms of investigating of the quiet and active solar atmosphere with the Ca II $854.2 \mathrm{~nm}$ line. For example, the high temporal cadence at which full spectral profiles can be sampled and the availability of an extended FOV make this instrument a very promising tool for studying small-scale activity such as reconnection-driven explosive events or small flares.

In the case of the quiet Sun, given the temperature sensitivity of the outer wings of the CaII $854.2 \mathrm{~nm}$, it would be possible to derive the precise run of temperature with height over an extended FOV, as a check on the realism of 3D hydrodynamical simulations of surface convection in the upper photosphere. Even more interesting, such data should be well-suited to investigating the presence and relevance of waves at different layers in the photosphere (Rutten et al. 2004; Janssen \& Cauzzi 2006; Wöger et al. 2006). Information on the spectral behavior of the line core, acquired at high spatial resolution over extended periods of time, should be able to shed light on several unresolved issues related to the chromospheric structure, such as the occurrence and relevance of acoustic shocks. While it is commonly accepted that acoustic waves at frequencies above the acoustic cutoff produce shocks in the quiet chromosphere, it is still hotly debated to what extent this kind of waves can contribute to the general chromospheric heating (Fossum \& Carlsson 2005; Wedemeyer-Böhm et al. 2007) and what role they play in the overall chromospheric structuring (Avrett 2007). Even more pressing would be an understanding of the role of acoustic waves and shocks in and around magnetic elements, especially those composing the "quiet" network. Several recent papers (De Pontieu et al. 2004; Hansteen et al. 2006; Jefferies et al. 2006) have in fact addressed the possibility that small scalemagnetic elements might channel part of the powerful, lowfrequency photospheric $p$-modes into higher atmospheric layers where they can develop into shocks and structure the surrounding environment. As described in the Introduction, the use of a 2D spectrometer in this case offers a big advantage over fixedslit spectrographs, both because of the possibility of following a posteriori the wandering of the magnetic elements during the course of the observations, and of the availability of an extended FOV. The latter supplies a greatly improved statistics, as well as important information on the local magnetic topology.

In forthcoming papers using IBIS Ca II $854.2 \mathrm{~nm}$ observations, we will demonstrate that acoustic shocks are a pervasive characteristic of the quiet chromosphere, even at the relatively low layers sampled by this line (Vecchio et al. 2007a) and that indeed there is spectral evidence of strong shocks of an acoustic origin around quiet magnetic network elements (Cauzzi et al., in preparation). In both cases the presence of the ubiquitous fibrils discussed in Sects. 5.3 and 5.4 proves to be a key ingredient in the chromospheric structure, outlining the magnetic canopy and defining atmospheric volumes with very different dynamical properties (Vecchio et al. 2007b). This strongly supports the remarks of Rutten (2007) that a full understanding of the chromosphere cannot develop without addressing its 3D nature. A comprehensive approach is needed that includes forward 2D and 3D numerical simulations coupled with detailed radiative transfer, on the one side, and high resolution (temporal, spatial and spectral) observations over extended fields of view, on the other. The latter task is currently best addressed by high-performance imaging devices adopting rapidly tunable Fabry-Perot interferometers such as IBIS, which eventually will be able also to provide full spectro-polarimetric observations.

Indeed, although we have not addressed the issue in this paper directly, the Ca II $854.21 \mathrm{~nm}$ line is probably the most reliable spectral line for measuring magnetic field strength and orientation in the solar chromosphere. In fact, its contribution function (Fig. 5) suffers much less from the formation gap around the temperature minimum than that of $\mathrm{H} \alpha$. As a result, response functions of the 854.2 Stokes $V$ profile to perturbations in the magnetic field (Uitenbroek 2006a) indicate that Zeeman polarization in the line would be much more sensitive to the chromospheric field, and would be so over a more coherent range of heights than for $\mathrm{H} \alpha$ (Socas-Navarro \& Uitenbroek 2004). Moreover, because hydrogen is a much lighter element and has a large Doppler width, the latter line has intrinsically low linear polarization from the transverse Zeeman effect, which is proportional to the square of the ratio of Zeeman splitting over Doppler width (Stenflo 1994, p. 259). The Ca II H and K lines are less suitable for magnetometry because they require modeling with partial frequency redistribution (PRD), and the precise interaction of PRD and Zeeman polarization is theoretically not wellestablished. The NaI D lines only provide magnetic sensitivity in the upper photosphere and lower chromosphere (Uitenbroek 2006a).

Interpretation of polarized spectra from the the CaII 854.2 nm line, as well as other chromospheric signatures, will require full inversion techniques, because of the wide range in formation heights over the width of the lines. One such technique has been explored to determine the chromospheric magnetic field in sunspots (Socas-Navarro et al. 2000; Socas-Navarro 2005) and in the quiet Sun (Pietarila et al. 2007a), showing promise that, with high resolution spectro-polarimetric imaging such as those provided by IBIS, significant progress can be made in the determination of chromospheric magnetic fields.

Acknowledgements. We want to remember here our friend and colleague B. Caccin, prematurely deceased in June 2004, with whom we exchanged many discussions about the adoption of Ca II $854.2 \mathrm{~nm}$ in IBIS. His wisdom and insight are deeply missed. This paper benefitted from discussion with V. Andretta, B. Fleck, M. Carlsson, R. J. Rutten, and A. Tritschler. The continuous help and patience of the observers M. Bradford, J. Elrod, and D. Gilliam at the DST are greatly appreciated. IBIS was built by INAF/Osservatorio Astrofisico di Arcetri with contributions from the Università di Firenze and the Università di Roma "Tor Vergata". IBIS construction and operation has been supported by the Italian Ministero dell'Università e della Ricerca (MUR), as well as the Italian Ministry of Foreign Affairs (MAE). NSO is operated by the Association of Universities for Research in Astronomy, Inc. (AURA), under cooperative agreement with the National Science Foundation. This research was supported through the European Solar Magnetism Network (ESMN, contract HPRN-CT-2002-00313), grant PRIN 2004 MIUR, and the Italian Ministry of Foreign Affairs (MAE). 


\section{Appendix A: Captions for the online movies}

Movie 1: IBIS spectral scan within the blue wing of the Ca II $854.2 \mathrm{~nm}$ line. FOV is about $60^{\prime \prime} \times 40^{\prime \prime}$, with spatial scale of $0.082^{\prime \prime} /$ pixel. The first image shows the solar scene acquired with a co-temporal G-band channel and displays normal granulation around two small pores and a weak plage region. The spectral images span from $65 \mathrm{pm}$ in the blue wing of the Ca II 854.2 to line core, in steps of $4 \mathrm{pm}$. The movies then fades from the core image back to the G-band image. Each monochromatic image has been obtained via speckle-reconstruction (Wöger 2006) of a burst of 50 images acquired within $20 \mathrm{~s}$. The sequence of narrowband images illustrates how by sampling the line from wing to core one gradually samples higher layers of the atmosphere, from the upper photosphere (where reversed granulation appears obvious) to the chromosphere dominated by fibrillar structures.

Movie 2: Temporal evolution of the line-core intensity of Ca II $854.2 \mathrm{~nm}$, for the data of May 31, 2004. The spatial scale is in arcsec. The movie spans $55 \mathrm{~min}$, with a time step of $19 \mathrm{~s}$ (175 images). See text for discussion.

\section{References}

Andretta, V., Busà, I., Gomez, M. T., \& Terranegra, L. 2005, A\&A, 430, 669 Avrett, E. H. 2007, in The Physics of Chromospheric Plasmas, ed. P. Heinzel, I. Dorotovič, \& R. J. Rutten, ASP Conf. Ser., 368, 81

Beckers, J. M., Mauter, H. A., Mann, G. R., \& Brown, D. R. 1972, Sol. Phys., 25,81

Carlsson, M., \& Stein, R. F. 1995, ApJ, 440, L29

Carlsson, M., \& Stein, R. F. 1997, ApJ, 481, 500

Cauzzi, G., Reardon, K. P., Vecchio, A., Janssen, K., \& Rimmele, T. 2007, in The Physics of Chromospheric Plasmas, ed. P. Heinzel, I. Dorotovič, \& R. J. Rutten, ASP Conf. Ser., 368, 127

Cavallini, F. 2006, Sol. Phys., 236, 415

Cheung, M. C. M., Schüssler, M., \& Moreno-Insertis, F. 2007, A\&A, 461, 1163

Chmielewski, Y. 2000, A\&A, 353, 666

De Pontieu, B., Erdélyi, R., \& James, S. P. 2004, Nature, 430, 536

De Pontieu, B., Hansteen, V. H., Rouppe van der Voort, L., van Noort, M., \& Carlsson, M. 2007, ApJ, 655, 624

Deubner, F.-L. \& Fleck, B. 1990, A\&A, 228, 506

Eddy, J. A. 1973, Sol. Phys., 29, 23

Fleck, B., \& Deubner, F.-L. 1989, A\&A, 224, 245

Fleck, B., Deubner, F.-L., Hofmann, J., \& Steffens, S. 1994, in Chromospheric Dynamics, ed. M. Carlsson, 103

Fontenla, J. M., Avrett, E. H., \& Loeser, R. 1993, ApJ, 406, 319

Fossum, A., \& Carlsson, M. 2005, Nature, 435, 919

Hansteen, V. H., De Pontieu, B., Rouppe van der Voort, L., van Noort, M., \& Carlsson, M. 2006, ApJ, 647, L73

Harvey, J. W. 2005, AGU Spring Meeting Abstracts, B5

Janssen, K., \& Cauzzi, G. 2006, A\&A, 450, 365

Janssen, K., Vögler, A., \& Kneer, F. 2003, A\&A, 409, 1127

Jefferies, S. M., McIntosh, S. W., Armstrong, J. D., et al. 2006, ApJ, 648, L151

Judge, P. 2006, in Solar MHD Theory and Observations: A High Spatial Resolution Perspective, ed. J. Leibacher, R. F. Stein, \& H. Uitenbroek, ASP Conf. Ser., 354, 259

Judge, P. G., Tarbell, T. D., \& Wilhelm, K. 2001, ApJ, 554, 424

Kalkofen, W. 1997, ApJ, 486, L145

Langangen, O., Carlsson, M., Rouppe van der Voort, L., Hansteen, V., \& De Pontieu, B. 2008, ApJ, 673, 1194

Leenaarts, J., Rutten, R. J., Carlsson, M., \& Uitenbroek, H. 2006, A\&A, 452, L15

Linsky, J. L., Teske, R. G., \& Wilkinson, C. W. 1970, Sol. Phys., 11, 374
Linsky, J. L., Hunten, D. M., Sowell, R., Glackin, D. L., \& Kelch, W. L. 1979, ApJS, 41, 481

Lites, B. W. 1984, ApJ, 277, 874

Lites, B. W., Rutten, R. J., \& Kalkofen, W. 1993, ApJ, 414, 345

Lyot, B. 1933, C. R. Acad. Sci., Paris, 197, 1593

Mein, P. 1971, Sol. Phys., 20, 3

Mein, P. 1991, A\&A, 248, 669

Mein, P. 2002, A\&A, 381, 271

Mein, P., Briand, C., Heinzel, P., \& Mein, N. 2000, A\&A, 355, 1146

Noyes, R. W. 1967, in Aerodynamic Phenomena in Stellar Atmospheres, ed. R. N. Thomas, IAU Symp., 28, 293

Pasachoff, J. M., Noyes, R. W., \& Beckers, J. M. 1968, Sol. Phys., 5, 131

Pietarila, A., Socas-Navarro, H., Bogdan, T., Carlsson, M., \& Stein, R. F. 2006, ApJ, 640, 1142

Pietarila, A., Socas-Navarro, H., \& Bogdan, T. 2007a, ApJ, 670, 885

Pietarila, A., Socas-Navarro, H., \& Bogdan, T. 2007b, ApJ, 663, 1386

Puschmann, K. G., Kneer, F., Seelemann, T., \& Wittmann, A. D. 2006, A\&A, 451,1151

Qu, Z.-Q., \& Xu, Z. 2002, Chin. J. Astron. Astrophys., 2, 71

Reardon, K. P., \& Cavallini, F. 2007, A\&A, in press

Reardon, K. P., Cauzzi, G., \& Rimmele, T. 2007, in The Physics of Chromospheric Plasmas, ed. P. Heinzel, I. Dorotovič, \& R. J. Rutten, ASP Conf. Ser., 368, 151

Rimmele, T. R. 2004, in Advancements in Adaptive Optics, Presented at the Society of Photo-Optical Instrumentation Engineers (SPIE) Conf., ed. D. B. Calia, B. L. Ellerbroek, \& R. Ragazzoni, Proc. SPIE, 5490, 34

Rouppe van der Voort, L. H. M., De Pontieu, B., Hansteen, V. H., Carlsson, M., \& van Noort, M. 2007, ApJ, 660, L169

Rutten, R. J. 2006, in Solar MHD Theory and Observations: A High Spatial Resolution Perspective, ed. J. Leibacher, R. F. Stein, \& H. Uitenbroek, ASP Conf. Ser., 354, 276

Rutten, R. J. 2007, in The Physics of Chromospheric Plasmas, ed. P. Heinzel, I. Dorotovič, \& R. J. Rutten, ASP Conf. Ser., 368, 27

Rutten, R. J., \& Uitenbroek, H. 1991, Sol. Phys., 134, 15

Rutten, R. J., de Wijn, A. G., \& Sütterlin, P. 2004, A\&A, 416, 333

Shine, R. A., \& Linsky, J. L. 1972, Sol. Phys., 25, 357

Shine, R. A., \& Linsky, J. L. 1974, Sol. Phys., 39, 49

Smith, G., \& Drake, J. J. 1987, A\&A, 181, 103

Socas-Navarro, H. 2005, ApJ, 631, L167

Socas-Navarro, H., \& Uitenbroek, H. 2004, ApJ, 603, L129

Socas-Navarro, H., Trujillo Bueno, J., \& Ruiz Cobo, B. 2000, ApJ, 530, 977

Socas-Navarro, H., Elmore, D., Pietarila, A., et al. 2006, Sol. Phys., 235, 55

Stenflo, J. O., ed. 1994, Astrophysics and Space Science Library, 189, Solar magnetic fields: polarized radiation diagnostics

Title, A. M. 1966, Mount Wilson and Palomar Observatories, Pasadena

Tritschler, A., Schmidt, W., Langhans, K., \& Kentischer, T. 2002, Sol. Phys., 211,17

Tziotziou, K., Tsiropoula, G., \& Mein, P. 2002, A\&A, 381, 279

Tziotziou, K., Tsiropoula, G., Mein, N., \& Mein, P. 2006, A\&A, 456, 689

Uitenbroek, H. 1989, A\&A, 213, 360

Uitenbroek, H. 2006a, in Solar MHD Theory and Observations: A High Spatial Resolution Perspective, ed. J. Leibacher, R. F. Stein, \& H. Uitenbroek, ASP Conf. Ser., 354, 313

Uitenbroek, H. 2006b, ApJ, 639, 516

Uitenbroek, H., Balasubramaniam, K. S., \& Tritschler, A. 2006, ApJ, 645, 776

van Noort, M. J., \& Rouppe van der Voort, L. H. M. 2006, ApJ, 648, L67

van Noort, M., Rouppe van der Voort, L., \& Löfdahl, M. G. 2005, Sol. Phys., 228,191

Vecchio, A., Cauzzi, G., \& Reardon, K. P. 2007a, A\&A, to be submitted

Vecchio, A., Cauzzi, G., Reardon, K. P., Janssen, K., \& Rimmele, T. 2007b, A\&A, 461, L1

Wedemeyer-Böhm, S., Steiner, O., Bruls, J., \& Rammacher, W. 2007, in The Physics of Chromospheric Plasmas, ed. P. Heinzel, I. Dorotovič, \& R. J. Rutten, ASP Conf. Ser., 368, 93

Wöger, F. 2006, Ph.D. Thesis, Albert-Ludwigs Universität Freiburg, Freiburg, Germany

Wöger, F., Wedemeyer-Böhm, S., Schmidt, W., \& von der Lühe, O. 2006, A\&A, 459, L9 